\title{
Cooperativas y SAT agroaimentarasas una comparación de la calidad del resultado contable
}

\author{
María Isabel Juárez Rubio, Maribel Leiva Jurac, \\ Pere Sabaté Prats y Óscar Alfranca Buriel
}

\section{RESUMEN}

Las empresas asociativas agroalimentarias son instituciones importantes a la hora de reforzar la posición económica de los agricultores y de promover el desarrollo regional y el empleo rural. No obstante, para fomentar el desarrollo de actividades económicas y sociales necesitan adoptar importantes medidas de modernización, de gestión empresarial y de comercialización. La calidad de la información contable es básica para las empresas, pues facilita la toma de decisiones y mejora las posibilidades en la captación de recursos financieros.

El objetivo de este artículo es explorar y comparar la calidad de la información contable presentada por las cooperativas y las SAT agroalimentarias en la última década y conocer qué grupo ha proporcionado una información financiera de mayor calidad.

PALABRAS CLAVE: Cooperativas y SAT agroalimentarias, calidad de la información contable, atributos de la calidad del resultado contable.

CLAVES ECONLIT: Q130, M410, M490.

Cómo citar este artículo: JUÁREZ, M.I, LEIVA, M., SABATÉ, P. \& ALFRANCA, O. (2015): "Cooperativas y SAT agroalimentarias: una comparación de la calidad del resultado contable", CIRIEC-España, Revista de Economía Pública, Social y Cooperativa, 84, 229-250.

Correspondencia: M. Isabel Juárez, Dpto. AEGERN, Universidad de Lleida, mjuarez@aegern.udl.cat. 


\section{Coopératives et SAT (sociétés agricoles de production) agroalimentaires : comparaison de la qualité du résultat comptable}

RÉSUMÉ : Les entreprises associatives agroalimentaires sont des institutions importantes au moment de renforcer la position économique des agriculteurs et de promouvoir le développement régional et l'emploi rural. Cependant, pour encourager le développement d'activités économiques et sociales, elles doivent adopter d'importantes mesures de modernisation, de gestion d'entreprise et de commercialisation. La qualité de l'information comptable est essentielle pour les entreprises car elle facilite la prise de décisions et améliore les possibilités de captation de ressources financières.

L'objectif de cet article est d'explorer et de comparer la qualité de l'information comptable présentée par les coopératives et par les SAT (sociétés agricoles de production) agroalimentaires au cours des dix dernières années et de savoir quel groupe a fourni une information financière de meilleure qualité.

MOTS CLÉ : Coopératives et SAT (sociétés agricoles de production) agroalimentaires, qualité de l'information comptable, attributs de la qualité du résultat comptable.

\section{Agri-food cooperatives and SATs: a comparison of the quality of accounting earnings}

ABSTRACT: Associative agri-food enterprises are important in strengthening the economic position of farmers and promoting regional development and rural employment. However, to encourage the development of economic and social activities they need to adopt important modernisation, business management and marketing measures. The quality of accounting information is essential for businesses, as it facilitates decision-making and improves their chances of attracting financial resources.

The aim of this article is to explore and compare the quality of the accounting information provided by cooperatives and agri-food SATs in the last decade and discover which group has provided higher-quality financial information.

KEY WORDS: Agri-food cooperatives, accounting quality, earnings attributes. 


\section{1.- Introducción*}

Las empresas de economía social juegan un papel esencial sobre el territorio al fomentar el desarrollo regional y el empleo rural (Juliá y Server, 2003; Juliá y Meliá, 2008), contribuyendo al proceso de recuperación económica. Son instituciones importantes a la hora de reforzar la posición económica de los agricultores, participando en negociaciones con las empresas ofertantes de insumos agrícolas o con los compradores de producción agrícola. En las últimas décadas, su crecimiento empresarial ligado a la necesidad de atraer capital social adicional, ha propiciado modificaciones importantes en la estructura de gobierno de estas empresas en Europa (Nilsson, 1999; Bijman, 2013).

Para fomentar el desarrollo de actividades económicas y sociales dichas entidades necesitan adoptar importantes medidas de modernización, de gestión empresarial y de comercialización. Llevar adelante estas medidas requiere disponer de suficientes recursos financieros, y el acceso a la financiación y su coste están ligados a una información contable de calidad.

Por tanto, la calidad de la información contable es básica para las empresas, pues facilita la toma de decisiones y mejora las posibilidades en la captación de recursos financieros. Dicha calidad en la información es apreciada tanto por socios como por acreedores ya que reduce la asimetría en la información, aumenta la transparencia y mejora las posiciones de negociación y contratación de la empresa (Watt y Zimmerman, 1986).

En España las entidades asociativas agroalimentarias están representadas por las Cooperativas y las Sociedades Agrarias de Transformación (SAT). La característica distintiva básica de dichas entidades es que mientras las SAT son empresas tanto personalistas como capitalistas, las cooperativas son sólo personalistas, lo que conduce a que sus diferencias se materialicen en su régimen económico (Román Cervantes, 2008). Existe la idea general que cooperativas y SAT son dos fórmulas organizativas distintas en su concepción jurídica, pero no tan diferentes en cuanto a las pautas de su comportamiento empresarial (Bel Durán, 1995). Se sabe poco de las SAT (aunque en algunas CC. AA. y en ciertos sectores éstas tienen un peso económico similar a las cooperativas) ya que han estado eclipsadas por el peso económico de las cooperativas y ello queda reflejado en los escasos estudios de análisis comparado entre cooperativas y SAT desde el punto de vista de la gestión empresarial (Vidal, et al., 2001; Galdeano Gómez y Rodríguez Rodríguez, 2000).

El interés de nuestro estudio gira en torno a cuán informativa es la documentación financiera presentada por estas empresas asociativas dividas en SAT y en cooperativas. El objetivo es comparar la calidad de la información contable presentada en la última década por cooperativas y SAT agroali-

* Los autores agradecen los comentarios de los evaluadores anónimos. 
mentarias y conocer si dicha calidad es similar en los dos grupos. Para ello utilizaremos cuatro contrastes empleados por la literatura de contabilidad positiva que permiten medir y comparar atributos como la calidad de los ajustes por devengo, la persistencia del resultado, la predictibilidad y el alisamiento del resultado. Cada uno de estos atributos representa propiedades específicas y deseables que proporcionan calidad al resultado empresarial (Lev, 1983; Penman y Zhang, 2002; Dechow y Dichev, 2002; Leuz et al., 2003; Francis et al., 2004; Cascino et al., 2010).

No hay estudios realizados con este enfoque sobre estas entidades asociativas en nuestro país. Sí que los hay con sociedades capitalistas cotizadas, pymes y con empresas familiares y no familiares. En dichos estudios, la calidad se ha estimado en base a medidas de resultado en distintos entornos: Gil de Albornoz e Illueca (2007) estudiaron la relación entre la calidad del resultado y el coste de la deuda de las pymes españolas; Bona Sánchez et al. (2007) concluyeron que las empresas de naturaleza familiar divulgan unos resultados de mayor calidad que las empresas no familiares; Callao et al.(2007) aportaron evidencias empíricas sobre la relación existente entre el gobierno y propiedad empresariales y la calidad de la información contable que presentan; Monterrey y Sánchez-Segura (2008) analizaron los efectos de las actividades de gobierno corporativo sobre la calidad del resultado de las compañías españolas cotizadas en Bolsa, e Iñiguez Sánchez y Poveda Fuentes (2008) estudiaron la calidad a través de la persistencia del resultado contable en tres países europeos.

El artículo se organiza de la forma siguiente: en el próximo apartado se hace una revisión del marco teórico; en el tercer apartado se presenta la muestra de empresas dividida en los dos grupos, se muestra el diseño de la investigación y se resume los resultados obtenidos. En el último apartado se presentan las conclusiones.

\section{2.- Marco teórico}

\subsection{Cooperativas y SAT. La calidad del resultado}

Es conocido que las cooperativas agroalimentarias son las entidades asociativas que mayor desarrollo empresarial han alcanzado, destacando claramente tanto en número de socios como en volumen de negocio: representan el 60\% del valor de toda la producción agrícola y ganadera de España, agrupan a más de un millón de socios y dan empleo a cerca de 100.000 personas en 2014, además de ser imprescindibles para la ordenación del territorio nacional y para la economía del medio rural. La presencia dominante de las cooperativas ha supuesto que, tradicionalmente, se considere a las SAT (12.511 empresas y 312.542 socios en 2013) como un mero apéndice del cooperativismo agrario con tendencia a transformarse en la fórmula cooperativa (Vidal et al., 2001). 
Las cooperativas se caracterizan por ser sociedades de propiedad colectiva que pertenecen a los beneficiarios de su actividad. Los socios de la cooperativa, además de ser propietarios y beneficiarios de las ganancias, pueden ser, incluso de forma simultánea, usuarios que venden y compran productos y/o servicios, trabajadores y directivos. Como consecuencia de estos roles diferentes que los socios adoptan en su relación con la cooperativa, los objetivos que persiguen pueden ser muy distintos, y no siempre compatibles (Nilsson, 1999).

En cuanto a la sociedad agraria de transformación, se trata de una fórmula de asociacionismo agrario peculiar del ordenamiento jurídico español: es una figura híbrida entre las sociedades civiles, cooperativas agrarias y sociedades mercantiles y que ha sido de gran utilidad para el desarrollo de ciertas zonas (Vargas Vasserot, 2010).

Las SAT como empresas sociales coinciden en líneas generales con los principios cooperativos, salvo en lo referente a las decisiones de implicación económica para los socios, ya que éstos pueden hacer uso de los votos que les corresponda en función de su participación. Asimismo, no les afecta los principios de libre adhesión y baja voluntaria, ni el de puertas abiertas ni es obligatorio el Fondo de reserva, Educación y Obras sociales. A pesar de ello, son empresas de economía social donde prima el interés del socio y no del capital (Juliá y Server, 2003; Román Cervantes, 2008).

Sin embargo, Vargas Vasserot (2010) considera que si bien es cierto que las SAT son sociedades participativas y esencialmente mutualistas, muchos aspectos de su régimen legal contradicen el espíritu de ese tipo de empresas, como por ejemplo, que el derecho de voto o los derechos económicos del socio se puedan ejercer en proporción directa al capital aportado, con lo que no se produce la primacía de la persona sobre el capital social ni el ideal democrático de este tipo de empresas ${ }^{1}$.

Por otro lado, la modificación e innovación de las estructuras tradicionales cooperativas en las últimas décadas (Nilsson, 1999; Bijman, 2013) implican reconsiderar su papel puramente social. Según Vargas y Aguilar (2004) la consideración "empresarial" de la cooperativa ha dejado de ser ocasional y marginal para hacerse "sistemática y habitual". Además, la aproximación de la cooperativa a la sociedad lucrativa ha sido posible gracias a la introducción de fórmulas de ingeniería organizativa y a la flexibilidad de los principios contables cooperativos (Vicent, 2002). Así mismo, en los últimos años los acontecimientos negativos conocidos en diferentes cooperativas europeas, tales como mala gestión, escándalos financieros y falta de democracia interna, han llevado a que la gobernanza cooperativa sea cuestionada (Cornforth, 2004) y ha demandado la necesidad mejorar las prácticas de información.

1.- Además, los operadores económicos que eligen estas sociedades están cómodos con su norma reguladora. Sobre todo porque si se compara con las de las cooperativas, son evidentes las ventajas que las SAT ofrecen ya que no tienen ninguna de las típicas limitaciones de las sociedades cooperativas, algunas derivadas de su peculiar estructura organizativa (dificultad para transmitir la posición de socio, límites de los derechos políticos y económicos de los socios inactivos, dificultad para la existencia de socios capitalistas), límites financieros (dificultad de concentrar capital social y atraer inversión externa, obligación de dotación de fondos de carácter irrepartible), límites operativos o funcionales (límites a las operaciones con terceros y límites para invertir en sociedades mercantiles) (Vargas Vasserot, 2010). 
Este trabajo sigue las investigaciones realizadas sobre la relación entre calidad de la información financiera y la estructura de gobernanza de la empresa. Tanto para cooperativas como para SAT, el papel de control del gobierno de la empresa sobre la calidad de la información se sitúa en el marco del problema de agencia derivado de la separación entre propiedad y gestión, que genera diferencias de intereses entre propietarios, gestores y acreedores (Jensen y Meckling, 1976; Álvarez Pérez et al., 2000; Arcas Larios, 2010). Además, la importancia de la información financiera en la fijación de contratos en la organización empresarial hace que la estructura contractual sea significativa en las políticas y prácticas contables adoptadas por la empresa (Watt y Zimmerman, 1986). En ocasiones, las diferencias de intereses llevan a desarrollar conductas oportunistas en las que la información contable juega un papel importante y en consecuencia puede convertirse en objeto de manipulación, lo que reduce la calidad de la misma.

El interés por evaluar la calidad de los informes financieros presentados por las empresas, y que éstos sean informativos y de utilidad para sus usuarios, ha interesado a la literatura contable desde hace tiempo. Aun así, en la literatura contable positiva el concepto de calidad de la información contable no tiene una definición única y para su medida se han utilizado distintos métodos (Revsine, 2002). Un amplio número de estudios valorando la calidad de la información contable ha examinado y aplicado algunas propiedades específicas del resultado, llamadas atributos del resultado como son, entre otros, la persistencia, la predictibilidad, etc. (Dechow et al., 2010).

De forma resumida, en cuanto a las medidas propuestas sobre cómo valorar la calidad del resultado (resultados previamente ponderados), algunos autores plantean la persistencia de los beneficios: los beneficios actuales pueden ser considerados un buen indicador de ganancias futuras. Otros presentan el resultado como de alta calidad si los beneficios son persistentes en el largo plazo (Lev, 1983; Penman y Zhang, 2002). En general, los conceptos aplicados de medición utilizados han sido la persistencia del resultado, la predictibilidad y el alisamiento del resultado.

Por otro lado, también se ha propuesto medir la calidad del resultado en función de la relación entre los ajustes por devengo y los flujos de caja. Recordar que el resultado contable es la medida que resume la eficacia de la empresa y que dicho resultado tiene dos componentes: el flujo de caja (FC) y los ajustes por devengo (AD). El primer componente es más objetivo y apenas manipulable por las políticas contables. El segundo depende en gran medida de la pericia y de la discrecionalidad de la gerencia y en ocasiones puede existir una manipulación de los informes contables con el objetivo de guiar las opiniones sobre los beneficios de la empresa (Dechow y Dichev, 2002). Todo ello repercute sobre la calidad del resultado y sobre la valoración y utilidad de la información publicada por las empresas. Así pues, otra manera de valorar la calidad de la información sería midiendo la calidad de los ajustes por devengo.

Hay numerosa investigación empírica centrada en dicha relación entre AD y FC y los factores que influyen en la misma: incertidumbre en el ámbito empresarial y económico, la habilidad del equipo de gestión, la discrecionalidad y manipulación de los AD por parte de la gerencia, etc. (Sloan, 1996; 
McNichols, 2002; Schipper y Vincent, 2003; Dechow et al., 2010). Dentro de esta corriente de investigación, una línea de trabajo ha girado en torno a la manipulación contable oportunista de los ajustes por devengo por parte de la gerencia con logros desiguales (Jones, 1991; Dechow et al., 2010; Kothari et al., 2005; McNichols, 2002; Francis et al., 2005). Para el caso español, García Osma et al. (2005; 2007), Monterrey y Sánchez-Segura (2008; 2009), Callao y Jarné (2011), entre otros, aportan evidencia empírica de este tipo de actuación.

El objetivo de este trabajo es explorar y comparar la calidad de la información contable suministrada por las cooperativas y por las SAT agroalimentarias en la última década. La hipótesis de trabajo -o conjetura- es que los dos grupos de empresas presentan una calidad de información contable similar, ya que la normativa que regula la constitución y estatutos de estas empresas, y la normativa contable a la que están sujetas son similares. Para contrastar esta hipótesis utilizaremos las medidas de calidad citadas previamente y que introducimos y aplicamos en el siguiente punto.

\section{3.- Diseño de la investigación y resultados}

\subsection{La selección de empresas y estadística descriptiva}

A partir de la base de datos $S A B I$, se ha buscado las empresas que cumplen con los requisitos de: forma jurídica cooperativas; empresas activas; pertenecientes a los sectores 01 (agricultura, ganadería y caza), 10 (industria de la alimentación), 11 (fabricación de bebidas) y a subsectores del 46 (4611, $4617,462,463)$ relacionados con el comercio y la intermediación de productos agropecuarios al por mayor (clasificación CNAE 2009). El total obtenido ha sido 2.198 entidades. Este número de empresas integra tanto cooperativas como SAT. Teniendo en cuenta la exigencia temporal del modelo que aplicamos en nuestro trabajo (Dechow y Dichev, 2002; Cascino et al., 2010), de este primer grupo inicial se seleccionaron las empresas que presentaran como mínimo 7 años de información financiera continuada, quedando dicha muestra inicial reducida a 80 empresas: 60 cooperativas y el resto SAT2 2 .

La distribución de empresas por sectores e ingresos de explotación se ha resumido en la Tabla 1.

2.- El periodo de años analizado incluye varios ejercicios económicos cuya contabilidad se regía por el PGC de 1990, y el período de 2008 a 2013, con las cuentas anuales elaboradas de acuerdo con las normas contables del nuevo PGC de 2007. En este trabajo, no se ha considerado el efecto que sobre los EEFF pueda haber tenido el cambio de normas contables. El motivo principal, ha sido la falta de información de Ios efectos económico-financieros de dicho cambio en la fuente de datos utilizada. En la base de datos SABI no figura detalle de dicha información. Tampoco se han considerado, por la misma razón, los efectos de los cambios de la Orden EHA/3360/2010 de 21 de diciembre, que modifica la Orden 3614/2003, de 16 de diciembre, de normas sobre aspectos contables de las sociedades cooperativas (Álvarez y Suárez 2012). Además, en este caso, por la complejidad que supone la existencia de una Ley estatal de Cooperativas y las diversas leyes autonómicas que regulan la actividad de las cooperativas, con peculiaridades diferenciadas. 


\section{Tabla 1. Distribución de las empresas por sectores y por ingresos de explotación}

\begin{tabular}{|c|c|c|c|c|c|c|c|c|c|}
\hline $\begin{array}{l}\text { Ingresos de Explotación } \\
\text { (miles de €) } 2013\end{array}$ & $\begin{array}{c}\text { Sector } \\
01\end{array}$ & $\begin{array}{c}\text { Sector } \\
10\end{array}$ & $\begin{array}{c}\text { Sector } \\
11\end{array}$ & $\begin{array}{c}\text { Sector } \\
46^{\star}\end{array}$ & COOPERAT. & $\begin{array}{c}\text { Sector } \\
01\end{array}$ & $\begin{array}{c}\text { Sector } \\
10\end{array}$ & $\begin{array}{c}\text { Sector } \\
46^{\star}\end{array}$ & SAT \\
\hline de 1.000 a 5.000 & 0 & 1 & 2 & 1 & 4 & & & & \\
\hline de 5.000 a 10.000 & 1 & & 1 & 5 & 7 & 1 & & & 1 \\
\hline de 10.000 a 50.000 & 4 & 5 & 3 & 28 & 40 & 1 & 3 & 10 & 14 \\
\hline de 50.000 a 100.000 & & 2 & & 2 & 4 & & & 3 & 3 \\
\hline Más de 100.000 & 1 & & & 4 & 5 & & & 2 & 2 \\
\hline Total & 6 & 8 & 6 & 40 & 60 & 2 & 3 & 15 & 20 \\
\hline
\end{tabular}

*Subsectores 4611, 4617, 462, 463

FUENTE: Elaboración propia a partir de datos SABI.

En la muestra con la que se ha trabajado, Tabla 1, el 70\% de empresas de cada grupo se localiza en el sector $46^{*}$. En relación con los ingresos de explotación, el $85 \%$ de las cooperativas y SAT superan los diez millones de euros en 2013; en el tramo de ingresos más alto, cinco cooperativas de segundo grado y dos SAT pasan de los cien millones de euros.

La Tabla 2 es una tabla de doble entrada, en la que se ordenan las empresas por intervalos de ingresos de explotación y de valores de fondos propios, activo y número de trabajadores. En el intervalo de ingresos de explotación de 10 a 50 millones de euros se sitúan el $55 \%$ de las cooperativas y el $60 \%$ de las SAT de la muestra.

En la Tabla 3 se muestran los valores de media, mediana y desviación típica del EBITDA, activo y fondos propios para el grupo más numeroso de empresas, tanto en cooperativas como en SAT, correspondiente al intervalo de ingresos de 10 a 50 millones de euros.

En la Tabla 4 se presentan los valores de media, desviación típica y mediana de las principales variables financieras tanto para cooperativas como para SAT. 


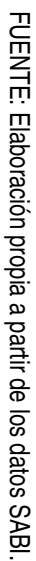

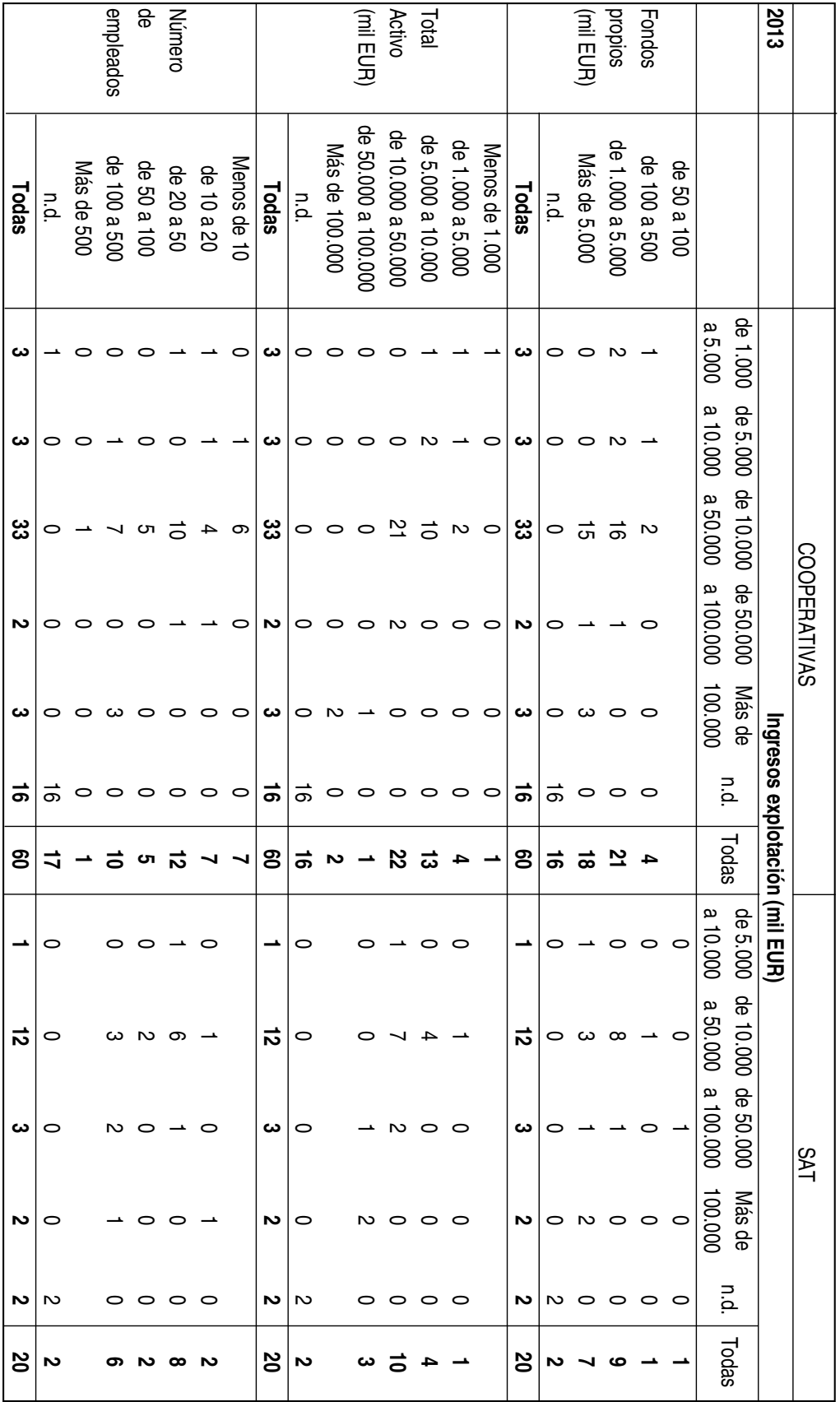


Tabla 3. Empresas del intervalo de ingresos de explotación de 10 a $50 \mathrm{M}$ de euros

\begin{tabular}{|l|ccc|ccc|}
\hline & \multicolumn{3}{|c|}{ 33 COOPERATIVAS } & \multicolumn{3}{c|}{ 12 SAT } \\
\hline & \multicolumn{3}{|c|}{ Ingresos de explotación (10.000 } & 50.000 mil EUR). Año 2013 \\
\hline (mil EUR) & Mediana & Desviación típica & Media & Mediana & Desviación típica & Media \\
\hline EBITDA & 447 & 444 & 593 & 834 & 753 & 1.057 \\
Activo & 11.694 & 7.107 & 13.278 & 13.090 & 6.510 & 13.531 \\
Fondos Propios & 4.766 & 3.894 & 5.451 & 3.961 & 2.901 & 4.217 \\
\hline
\end{tabular}

FUENTE: Elaboración propia a partir de los datos SABI.

\section{Tabla 4. Principales variables financieras por grupo de empresas}

\begin{tabular}{|l|ccc|ccc|}
\hline & \multicolumn{3}{|c|}{ COOPERATIVAS } & \multicolumn{3}{c|}{ SAT } \\
\hline Año 2013 & Media & Desviación típica & Mediana & Media & Desviación típica & Mediana \\
\hline ROA \% & 1,71 & 2,77 & 1,38 & 2,98 & 3,32 & 1,63 \\
RENTAB. EXPLOTACION \% & 4,99 & 3,83 & 4.27 & 6,69 & 4,07 & 6,15 \\
ENDEUDAMIENTO \% & 25,85 & 18,56 & 19,96 & 28,97 & 16,82 & 27,73 \\
COSTE MEDIO FINANC. EXTERNA & 0,31 & 1,66 & 0,05 & 0,04 & 0,03 & 0,03 \\
RATIO FM/ACTIVO & 0,12 & 0,16 & 0,07 & 0,14 & 0,14 & 0,13 \\
PM COBRO (DIAS) & 71,7 & 39,97 & 57,38 & 64,78 & 25,67 & 61,85 \\
GASTO PERSONAL/INGR. EXPLOT.\% & 6,04 & 9,22 & 2,91 & 5,52 & 3,33 & 4,83 \\
RATIO SOLIDEZ & 1,31 & 1,88 & 0,79 & 1,02 & 1,06 & 0,77 \\
RATIO LIQUIDEZ \% & 143,58 & 73,58 & 118,50 & 152,14 & 76,40 & 122,64 \\
R. ROTACION ACTIVOS & 1,94 & 1,36 & 1,55 & 2,35 & 0,95 & 2,08 \\
\hline
\end{tabular}

FUENTE: Elaboración propia a partir de los datos SABI.

\subsection{Medidas de la calidad del resultado contable}

Para capturar la extensión por la cual las empresas usan su discrecionalidad para hacer sus documentos anuales más o menos informativos, se necesitan medidas que valoren la calidad de dicha información.

La posible manipulación discrecional/oportunista de la información contable ha llevado a que las investigaciones realizadas sobre calidad giren en torno a un conjunto de proxies de medida denominadas atributos del resultado, y que representan propiedades específicas y deseables que propor- 
cionan calidad al resultado empresarial. Dichas propiedades están divididas en un primer grupo de atributos basado en información contable y un segundo grupo basado en datos del mercado bursátil (Dechow y Dichev, 2002; Francis et al., 2004; Cascino et al., 2010).

En nuestro caso y dado que son empresas no cotizantes, aplicamos el primer grupo de atributos basado en la contabilidad y que son: la calidad de los AD, la persistencia del resultado, la predictibilidad y el alisamiento del resultado. Dichas propiedades son utilizadas como variables de medida de la calidad del resultado.

\section{La calidad de los AD}

La calidad de los ajustes por devengo se ha definido en función de la relación entre AD y FC y los factores que influyen en esta relación, como son la incertidumbre del medio empresarial, la habilidad de la gerencia, la extensión en la manipulación de los devengos, etc. Para algunos autores este atributo es el más completo a la hora de medir la calidad del resultado (Francis et al. 2004; Dechow et al., 2010, Cascino et al. 2010).

Dechow y Dichev (2002) aportaron un nuevo enfoque a este atributo y que se ha generalizado en su aplicación: un resultado es de alta calidad si hay un importante grado de materialización de los $\mathrm{AD}$ en los FC, sin considerar los factores que puedan afectar a esta relación.

A la hora de contabilizar la realización anual de la empresa, el principio del devengo desvincula el registro de los ingresos y gastos del momento del cobro y pago de los mismos. Esta diferencia entre el FC y el beneficio contable se recoge en los ajustes por devengo (AD). Para estimar estos ajustes por devengo las normas contables otorgan a la gerencia una cierta dosis de discrecionalidad. Dechow y Dichev (2002) parten de la premisa que la calidad de los AD es el grado por el cual los AD de un año en concreto se materializa en los FC de dicho año y de los dos años adyacentes. Así pues, cuando la calidad es alta, la probabilidad de la materialización de los devengos en los FC es alta y esto muestra unos FC persistentes.

Por tanto, los $\mathrm{AD}$ acaban materializándose en los FC del período presente y de los inmediatamente adyacentes, por lo que AD correctamente calculados tienen un reflejo directo en la serie de FC generados por la empresa, mientras que aquellos que incorporan errores no se realizan en los FC y deben ser anulados. De esta manera, la calidad de los AD es menor cuanto mayor es la magnitud de los errores de los $A D$ no materializados. La desviación estándar de dichos errores del modelo es la proxy que mide la calidad del AD: empresas con desviaciones estándar mayores tienen devengos, FC y resultados más volátiles, y, por tanto, una menor calidad en la información suministrada (Dechow y Dichev, 2002).

La medida empírica que estas autoras proponen de la calidad del $A D$ a corto plazo es la proporción por la cual el capital circulante $(\triangle \mathrm{WC}$ ) se materializa en los flujos de caja de operaciones (FCO) y donde la falta de realizaciones significa baja calidad. El error se define como la diferencia entre la 
cantidad devengada y la cantidad realizada en períodos inmediatos. La medida de estimación de los errores de los $A D$ corresponde a los residuos de una serie de regresiones que recogen los cambios del capital circulante $(\triangle \mathrm{WC}$ ) sobre los FCO del año anterior, del presente y del siguiente. La desviación estándar de dichos residuos mide la calidad de los AD y, por tanto, la del resultado. $O$ de otro modo: la mayor desviación estándar de los residuos denota menor calidad de los AD (Gil de Albornoz e lllueca, 2007).

En este modelo, el capital circulante $(\triangle \mathrm{WC})$ y los FCO permiten centrarse en los $A D$ a corto plazo, en operaciones que transcurren dentro del año. Por tanto, no es aplicable a empresas cuyos ciclos de producción sean a largo plazo. Las empresas agroalimentarias que consideramos para nuestra investigación entrarían en la esta categoría. Como ya se ha comentado, otra limitación del modelo es que no distingue los factores empresariales y de gerencia que puedan influir en la relación FCO y WC.

Partiendo del modelo de Dechow y Dichev (2002), lo aplicamos con la extensión propuesta por McNichols (2002) que mejora el poder explicativo del modelo original (McNichols (2002); Francis et al., 2004; 2005). En la expresión EQ-1, la variación de los AD ( $\triangle W C$ ) a corto plazo es la variable dependiente y los FCO del ejercicio actual, anterior y siguiente, la variación de ventas y el inmovilizado material son las variables independientes. Todas las variables están ponderadas por el activo medio anual. Los residuos de la regresión reflejan los AD no materializados en los FCO. La desviación estándar de los residuos de la ecuación es la medida inversa de la calidad de los AD. La ecuación queda:

$$
\begin{aligned}
\Delta \mathrm{WC}_{i t} \mathrm{TA}_{i t}=B_{0} & +\beta_{1} \mathrm{FCO}_{-} \mathrm{TA} A_{i, t-1}+\beta_{2} \mathrm{FCO}_{-} \mathrm{TA}_{i t}+\beta_{3} \mathrm{FCO}_{-} \mathrm{TA}_{i, t+1}+\beta_{4} \Delta \mathrm{VENTAS}_{-} \mathrm{TA}_{i t} \\
& +\beta_{5} \text { INMOVILIZADO_TA }_{i t}+\sum \alpha_{i} \mathrm{ANO}_{i t}+\varepsilon_{i t}(\mathrm{EQ}-1)
\end{aligned}
$$

De donde:

- $\Delta \mathrm{WC}_{i t}=$ ajustes por devengo a corto plazo de la empresa $i$ en el año $t$, calculados como el cambio en activo corriente menos el cambio en la tesorería e inversiones financiera temporales, menos el cambio en pasivo corriente más el cambio en la deuda bancaria a corto plazo. El cambio en la variable correspondiente para los ejercicios $t-1$ y $t$.

- $\mathrm{FCO}_{i t-1}, \mathrm{FCO}_{i t}, \mathrm{FCO}_{i t+1}=$ flujo de caja operativo de la empresa i en los ejercicios $t-1, t$ y $t+1$ respectivamente, calculado como la diferencia entre el resultado de la actividad ordinaria (RAO) y los ajustes por devengo totales del ejercicio correspondiente $\left(A D_{i t}=\Delta W C_{i t}\right.$ - Amortizaciones $\left._{i t}\right)$.

- $\triangle$ VENTAS $_{i t}=$ cambio de la cifra de ventas de la empresa $i$ del ejercicio $t-1$ al $t$.

- INMOVILIZADO $i t=$ valor del inmovilizado material de la empresa $i$ en el año $t$.

- ANO $_{i t}=$ variable dummy anual

- _ $\mathrm{TA}_{i t}$, todas las variables están divididas por el activo total medio de la empresa $i$ en el año $t$, calculado como media aritmética del activo total en los años $t-1$ y $t$. 
Se utiliza la desviación estándar del residuo $(A D=\sigma(\varepsilon))$ como medida inversa de la calidad de los AD de la empresa correspondiente al conjunto del período considerado. Para obtener una medida de la calidad de los ajustes por devengo relativa al año concreto, Dechow y Dichev sugieren utilizar el valor absoluto del residuo en ese año.

\section{Persistencia}

La persistencia del resultado es otro de los atributos de la calidad pues captura la sostenibilidad de los resultados y los persistentes son considerados como deseables por su recurrencia (Lev, 1983; Revsine et al., 2002; Penman y Zhang, 2002).

Para estimar este indicador, seguimos a Lev (1983) que utiliza la expresión EQ-2:

$$
\mathrm{RAO}_{-} \mathrm{TA} i t=\beta_{0}+\beta_{1} \mathrm{RAO}_{-} \mathrm{TA} \mathrm{A}_{i, t-1}+\sum \alpha_{i} \mathrm{AN} \mathrm{O}_{i, t}+\varepsilon_{i t}(\mathrm{EQ}-2)
$$

Donde:

- RAO_TA $A_{i t}=$ es el resultado de la actividad ordinaria antes de impuestos de la empresa i en el año $t$, dividido por el activo medio anual

$$
\mathrm{AN} \mathrm{O}_{i, t}=\text { variable año dummy }
$$

La medida empleada para calcular la persistencia es el coeficiente $\beta_{1}$ : cuanto más próximo a la unidad, resultados más recurrentes y sostenibles en el tiempo, y por tanto mayor persistencia en los resultados.

Se considera que cuando el resultado es más sostenible, éste es un mejor indicador de los flujos de caja futuros y suministra una información más útil para los usuarios. Dicha persistencia permite la predicción y la investigación sobre qué factores hacen que el resultado sea una variable más fácil de predecir que la de los FC (Sloan, 1996; Revsine et al., 2002; Francis et al., 2005).

\section{Predictibilidad}

Este atributo facilita la habilidad de los analistas y usuarios de predecir futuras ganancias a partir de la trayectoria de las pasadas. Se valora la capacidad del resultado de predecirse a sí mismo. Dicha predictibilidad se mide como la raíz cuadrada de la varianza de los residuos $\left(\sigma^{2}\left(\varepsilon_{t}\right)\right)^{1 / 2}$ de la EQ-2. Valores altos en la raíz cuadrada de la varianza de los residuos implica menor predictibilidad en los resultados. (Francis et al., 2004). 


\section{Alisamiento}

El objetivo de practicar el alisamiento del resultado es que los usuarios de la información se sienten más confiados en la gestión cuando la gerencia es capaz de informar sobre resultados estables, y no tanto cuando hay fluctuaciones, ya que éstas se asocian a riesgos de la inversión (Apellániz Gómez, 1991).

El alisamiento se considera un atributo deseable de la calidad, ya que los gestores usan su información interna sobre futuros ingresos para alisar las fluctuaciones transitorias y por tanto lograr una información financiera más representativa y útil sobre los resultados presentados, reduciendo así la asimetría en la información (Demski, 1998; Ronen y Sadan, 1981).

Sobre este proceso de alisamiento existe un considerable cuerpo de literatura que sugiere que las empresas suavizan o gestionan sus beneficios a través del tiempo para reducir ciertos riesgos asociados a beneficios excesivamente volátiles (Dechow et al., 2010; Gabás y Pina, 1991). Esto significa que los beneficios no están sistemáticamente sesgados al alza, sino que la gestión del resultado tratará de atenuar el impacto negativo que sobre el beneficio pueden tener periodos difíciles y, a la vez, suavizar un posible efecto exagerado de buenos tiempos económicos.

Para el último atributo, el test de alisamiento que se utiliza es la ratio de la desviación estándar del resultado de actividad ordinaria dividido por la desviación estándar del FCO -tanto resultado de la actividad ordinaria y como FCO están ponderadas por el activo total medio, $\sigma\left(\mathrm{RAO}_{-} \mathrm{TA}\right)_{j, t} / \sigma\left(\mathrm{FCO} \_\mathrm{TA}\right)_{j, t}$ - (Leuz et al., 2003; Cascino et al., 2010). El método utilizado indica que valores altos de la ratio sugieren menor alisamiento de las ganancias.

\subsection{Resultados}

En el al Cuadro 1 se resume los resultados obtenidos de las regresiones de EQ-1 con las SAT y las cooperativas en relación con la calidad de los AD. El modelo se estima como un modelo de efectos fijos usando MCO donde se han controlado los efectos anuales introduciendo una variable ficticia que toma valor uno en el año de referencia y cero en el resto de casos. Tal y como predice la literatura (Dechow y Dichev, 2002; McNichols, 2002), los coeficientes para las tres variables FCO son menores que la unidad y de valor negativo para $\mathrm{FCO}_{t}$, indicando que los datos se adecuan al modelo teórico. En la muestra de las cooperativas, el signo para las variables $\triangle$ VENTAS e INMOVILIZADO coincide con los publicados por otros trabajos (McNichols, 2002; Francis et al. 2005), no así el signo de la variable INMOVILIZADO del grupo SAT que en nuestro caso es positivo.

Para contrastar si la calidad de los AD en los dos grupos es similar, comparamos la media y la mediana de las desviaciones estándar de los residuos $\left(\sigma\left(\varepsilon_{i t}\right)\right)$ de las regresiones por grupo de empresas y que capturan la capacidad de materialización de los AD en los FCO. Para los test de contraste 
de media se utiliza los test t paramétrico y para la mediana se utiliza el test no paramétrico MannWhitney. El nivel de significación utilizado es del de 5\% de significación.

\section{Cuadro 1. Calidad de los ajustes por devengo}

\begin{tabular}{|c|c|c|c|c|c|c|c|}
\hline \multicolumn{8}{|c|}{ 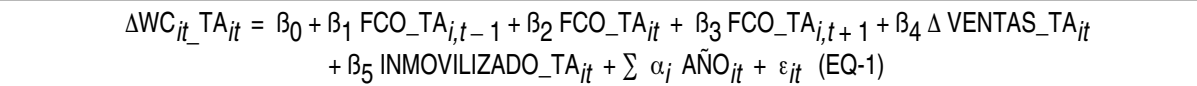 } \\
\hline \multicolumn{4}{|c|}{ COOPERATIVAS } & \multicolumn{4}{|c|}{ SAT } \\
\hline$\Delta \mathrm{WC}_{-} \mathrm{TA}_{t}$ & Coef. & $t$ & $P>|t|$ & $\Delta \mathrm{WC}_{-} \mathrm{TA}_{t}$ & Coef. & $t$ & $P>|t|$ \\
\hline $\mathrm{FCO}_{-} \mathrm{TA}_{t-1}$ & .1140 & 4.95 & 0.000 & $\mathrm{FCO}_{-} \mathrm{TA}_{t-1}$ & .0979 & 4.18 & 0.000 \\
\hline $\mathrm{FCO}_{-} \mathrm{TA}_{t}$ & -.6228 & -27.69 & 0.000 & $\mathrm{FCO}_{-} \mathrm{TA}_{t}$ & -.8309 & -36.04 & 0.000 \\
\hline $\mathrm{FCO}_{-} \mathrm{TA}_{t+1}$ & .1324 & 5.55 & 0.000 & $\mathrm{FCO}_{-} \mathrm{TA}_{t+1}$ & .1227 & 5.06 & 0.000 \\
\hline$\Delta$ VENTAS_TA $A_{t}$ & .0114 & 4.02 & 0.000 & $\Delta$ VENTAS_TA $_{t}$ & .0057 & 1.72 & 0.088 \\
\hline INMOVILI_TA $A_{t}$ & -.0097 & -0.57 & 0.566 & INMOVILI_TA & .0324 & 1.94 & 0.054 \\
\hline _cons & -.0231 & -0.35 & 0.728 & _cons & -.0336 & -1.20 & 0.233 \\
\hline \multirow{3}{*}{\multicolumn{4}{|c|}{$\begin{array}{l}\text { Efectos anuales, si } \\
\text { Number of obs. }=473 \\
\text { Adj R-squared }=0.7163\end{array}$}} & \multicolumn{4}{|c|}{ Efectos anuales, si } \\
\hline & & & & \multicolumn{4}{|c|}{ Number of obs. $=153$} \\
\hline & & & & \multicolumn{4}{|c|}{ Adj R-squared = 0.9284} \\
\hline
\end{tabular}

En el Cuadro 2 se presentan la media y la mediana de las desviaciones estándar de los residuos de la EC-1 por cooperativas y por SAT y el resultado del contraste. Se han obtenido valores menores tanto en media como en mediana para el grupo de SAT, lo que indica un mayor nivel de calidad de los AD en las SAT comparado con las cooperativas. Además, las diferencias en media y mediana entre los dos grupos son significativas, por lo que no se acepta la hipótesis nula de calidades similares.

\section{Cuadro 2. Calidad de los ajustes por devengo. Media y mediana de las desviaciones estándar de los residuos por cooperativas y por SAT}

\begin{tabular}{|lccc|}
\hline \multicolumn{4}{|c|}{ Calidad $\mathrm{AD}=\sigma\left(\varepsilon_{t}\right)$ de la ecuación EQ-1 } \\
\hline & Cooperativas & SAT & Contraste \\
\hline Media & .0360821 & .0232414 & $\mathrm{t}=3.862$ \\
Mediana & .0243766 & .0146464 & $\mathrm{z}=3.975$ \\
\hline
\end{tabular}

En resumen, la evidencia encontrada indicaría que las SAT tienen un nivel superior de calidad de $A D$ que las cooperativas. 
En relación con el atributo de la persistencia, el Cuadro 3 muestra los resultados de las regresiones en los dos grupos de empresas. La ecuación EQ-2 se estima como un modelo de efectos fijos usando MCO donde se han controlado los efectos anuales introduciendo una variable ficticia que toma valor uno en el año de referencia y cero en el resto de casos.

Siguiendo con el Cuadro 3 , el coeficiente $\beta_{1}$ de la variable RAO_TA $A_{t-1}$ mide la persistencia del resultado. Dicho coeficiente $\beta_{1}$ es superior en las cooperativas. Para comprobar si es significativa la diferencia de valor entre los dos grupos, se realiza un contraste de cambio estructural utilizando la muestra conjunta. Los resultados de este contraste se recogen en la tercera columna del Cuadro 3: el coeficiente de la variable COOPSAT ${ }^{*} \mathrm{RAO}_{-} \mathrm{TA}_{t-1}$ (que toma valor 1 para las cooperativas y cero para las SAT) es no significativo, por tanto, aceptamos la hipótesis nula de una persistencia de resultado similar en ambos grupos.

Por otro lado, los valores de $\mathrm{R}^{2}$ ajustado de las regresiones son similares a los obtenidos en otras publicaciones (Cascino et al., 2010). Como conclusión, los dos grupos presentan una calidad similar respecto al atributo de persistencia.

\section{Cuadro 3. Persistencia del resultado}

\begin{tabular}{|c|c|c|c|c|c|c|}
\hline \multicolumn{7}{|c|}{$\mathrm{RAO}_{-} \mathrm{TA}_{t}=\beta_{0}+\beta_{1} \mathrm{RAO}_{-} \mathrm{TA}_{t-1}+\sum \alpha_{i} \mathrm{AN}_{i, t}+\varepsilon_{t}(\mathrm{EQ}-2)$} \\
\hline & \multicolumn{2}{|c|}{ Cooperativas } & \multicolumn{2}{|c|}{ SAT } & \multicolumn{2}{|c|}{ Muestra conjunta } \\
\hline Parámetro & Coef. & $P>|t|$ & Coef. & $P>|t|$ & Coef. & $P>|t|$ \\
\hline Cons. & -.00454 & 0.828 & .01039 & 0.710 & -.00347 & 0.904 \\
\hline $\mathrm{RAO}_{-} \mathrm{TA}_{t-1}$ & .55406 & 0.000 & .52067 & 0.000 & .50674 & 0.000 \\
\hline COOPSAT* RAO_TA $_{t-1}$ & & & & & .05071 & 0.341 \\
\hline Efectos fijos anuales & $\mathrm{Si}$ & & $\mathrm{Si}$ & & $\mathrm{Si}$ & \\
\hline Number of obs. & 591 & & 280 & & 871 & \\
\hline Adj R-squared & 0.3779 & & 0.2904 & & 0.3232 & \\
\hline
\end{tabular}

En el Cuadro 4 se presenta los resultados obtenidos en relación con el atributo de predictibilidad, donde se ha calculado la media y mediana de la raíz cuadrada de la varianza de los residuos $\left(\sigma^{2}\left(\varepsilon_{t}\right)\right)^{1 / 2}$ de la regresión correspondiente a EQ-2, agrupados en cooperativas y SAT. Los valores obtenidos son menores para las cooperativas. Además la diferencia de media y de mediana en los dos grupos es significativa (t test paramétrico y no paramétrico Mann-Whitney al nivel del $5 \%$ de significación) por lo que rechazamos la hipótesis nula. Se concluye que las cooperativas muestran mayor predictibilidad de resultados que las SAT. 


\section{Cuadro 4. Predictibilidad del resultado}

\begin{tabular}{|c|c|c|c|}
\hline \multicolumn{4}{|c|}{$\begin{array}{c}\text { RAO_TA }_{t}=\beta_{0}+\beta_{1} \mathrm{RAO}_{-} \mathrm{TA}_{t-1}+\sum \alpha_{i} \mathrm{AÑO}_{i, t}+\varepsilon_{t}(\mathrm{EQ}-2) \\
\left(\sigma^{2}\left(\varepsilon_{t}\right)\right)^{1 / 2} \text { de la ecuación EQ-2 }\end{array}$} \\
\hline & Cooperativas & SAT & Contraste \\
\hline Media & .016474 & .029830 & $t=3.469$ \\
\hline Mediana & .014046 & .021517 & $z=3.522$ \\
\hline
\end{tabular}

Para el último atributo, Cuadro 5, el test de alisamiento que se utiliza es la ratio de la desviación estándar del resultado de actividad ordinaria y la desviación estándar del FCO. Para el cálculo se ha empleado una muestra flotante que permite aumentar el número de observaciones e incrementar el poder estadístico de cada test ("rolling 3-year-window") (Cascino et al., 2010).

En el Cuadro 5 se muestra que el valor de la media es menor en las SAT, y mayor el de la mediana. Según los test de contraste de media y mediana realizados (test t paramétrico y no paramétrico MannWhitney, nivel de significación del $5 \%$ ), las diferencias en los valores observados entre los dos grupos no son estadísticamente significativas, indicando que ambos grupos de empresas tienden a informar con un alisamiento del resultado similar.

\section{Cuadro 5. Resultados del test de alisamiento}

\begin{tabular}{|c|c|c|c|}
\hline \multicolumn{4}{|c|}{$\sigma\left(\mathrm{RAO}_{-} \mathrm{TA}_{j, t}\right) / \sigma\left(\mathrm{FCO}_{-} \mathrm{TA}_{j, t}\right)$} \\
\hline & Cooperativas & SAT & Contraste \\
\hline Media & .32555 & .31088 & $t=-.273$ \\
\hline Mediana & .11396 & .16642 & $z=.585$ \\
\hline
\end{tabular}




\section{4.- Conclusiones}

Un objetivo necesario para las empresas que compiten por los recursos en los mercados financieros es suministrar información contable de calidad. La contabilidad de calidad proporciona mayor información sobre las características de las actuaciones económicas de la empresa y es relevante para la toma de decisiones de los agentes relacionados con la actividad empresarial.

Este trabajo sigue las investigaciones realizadas sobre la relación entre calidad de la información financiera y la estructura de gobernanza de la empresa. El ámbito específico de investigación son las cooperativas y SAT agroalimentarias, básicas para el desarrollo y equilibrio rural, y para las cuales el proceso de competitividad y globalización exigirá ajustes de organización y una mayor calidad y transparencia de la información contable.

El papel de control del gobierno de la empresa sobre la calidad de la información, tanto para cooperativas como para SAT, se sitúa en el marco del problema de agencia, ya que hay intereses no coincidentes -a veces contrapuestos- entre los agentes interesados en el resultado de la empresa. En ocasiones, dichos intereses llevan a desarrollar conductas oportunistas donde la información contable juega un papel importante y puede verse afectada en su calidad.

El objetivo de este trabajo es comparar la información contable presentada en la última década por una muestra de cooperativas y SAT agroalimentarias y conocer si los dos grupos han proporcionado información financiera de calidad similar.

Nuestra hipótesis de partida es que las diferencias que establece la normativa que regula la constitución y los estatutos de estas empresas, así como la normativa contable a la que están sujetas, no deberían ser motivo para que la calidad de la información contable presentara diferencias significativas entre los dos tipos de sociedades. Para ello se han utilizado cuatro atributos empleados por la literatura de contabilidad positiva que permiten medir y comparar propiedades tales como la calidad de los ajustes por devengo, la persistencia del resultado, la predictibilidad y el alisamiento del resultado. Cada uno de estos atributos representa propiedades específicas y deseables que proporcionan una medida de la calidad al resultado empresarial.

En la muestra estudiada, la evidencia empírica indica que los dos grupos presentan una persistencia similar en el resultado y tienden a informar con un alisamiento de resultado parecido. Sin embargo, se han encontrado diferencias significativas en los dos atributos restantes. En el test de predictibilidad, los valores obtenidos indican que las cooperativas muestran una predictibilidad de resultados mayor que la revelada en las SAT. En el caso del atributo de calidad de los ajustes por devengo $(A D)$, la evidencia de la muestra indica un mayor nivel de calidad de AD en las SAT que el suminis- 
trado por las cooperativas, siendo dicha diferencia significativa entre los dos grupos. Así pues, las SAT presentan menores desviaciones en la materialización de los AD en los flujos de caja operativos que las mostradas en las cooperativas. Este resultado indica una mejor realización de los AD por parte de las SAT.

En resumen, los resultados de la investigación realizada con los cuatro contrastes indican que no hay diferencias significativas en dos de ellos, pero que si las hay en los otros dos, lo que sugiere la necesidad de realizar futuras investigaciones con muestras de mayor tamaño que podrían aportar mayor evidencia sobre el tema estudiado.

\section{Bibliografía}

ÁLVAREZ PÉREZ, B., ARBESU LÓPEZ, P. \& FE CANTO, C. (2000): "Las cooperativas en el marco de la teoría de la agencia", CIRIEC-España, Revista de Economía Pública, Social y Cooperativa, 34, 169-188.

ÁLVAREZ PÉREZ, B. \& SUÁREZ ÁLVAREZ, E. (2012): "El resultado según las nuevas normas sobre aspectos contables de las sociedades cooperativas", Partida doble, 239, 36-49.

APELLÁNIZ GÓMEZ, P. (1991): "Una aproximación empírica al alisamiento de beneficios en la banca española", Revista española de financiación y contabilidad, XXI (66), 195-219.

ARCAS LARIO N. (2011): El gobierno de las cooperativas agroalimentarias. Factores de éxito, Colección Economía. Fundación Cajamar

BARTH, M.E., BEAVER, W.H. \& LANDSMAN, W.R. (2001): "The relevance of the value relevance literature for financial accounting standard setting: Another view", Journal of Accounting and Economics, 31, 77-104.

BEAVER, W., RICHARD, A. \& RYAN, S. (1987): "The information content of security prices: A second look", Journal of Accounting and Economics, 9, 139-157.

BEL DURAN, P. (1995): "Similitudes y diferencias entre las sociedades cooperativas agraria y las sociedades agrarias de transformación a la luz de los principios cooperativos tras el congreso de Manchester", REVESCO, Revista de Estudios Cooperativos, 61, 107-125

BIJMAN, J. (2013): "Las cooperativas agroalimentarias en la UE. Empresas modernas con retos modernos", Mediterráneo Económico, 24, 41-60.

BONA SÁNCHEZ, C., PÉREZ ALEMÁN, J. \& SANTANA MARTíN, D. (2007): "Family control and earnings quality", RC-SAR, 10 (1), 11-34. 
BUSHMAN, R.M. \& SMITH, A.J. (2001): "Financial accounting information and corporate governance", Journal of Accounting and Economics, 32, 237-333.

CALLAO GASTÓN, S., GASCA GALÁN, M. \& JARNE JARNE, J.I. (2007): "Corporate governance and financial reporting quality", RC-SAR, 10 (1), 133-156.

CALLAO GASTÓN, S. \& JARNE JARNE, J.I. (2011): "The impact of the crisis on earnings management", RC-SAR, 14 (2), 59-85.

CASCINO, S., PUGLIESE, A., MUSSOLINO, D. \& SANSONE, C. (2010): "The influence of family ownership on the quality of accounting information", Family Business review, 23, 246-265.

CORNFORTH, C. (2004): "The governance of cooperatives and mutual associations: a paradox perspective", Annals of Public and Cooperative Economics, 75(1), 11-32.

DECHOW, P.M. (1994): "Accounting Earnings and Cash Flows as Measures of Firm Performance: the Role of Accounting Accruals", Journal of Accounting \& Economics, 18(1), 3-42.

DECHOW, P.M. \& DICHEV, I.D. (2002): "The quality of accruals and earnings: The role of accrual estimation errors", Accounting Review, 77 (Suppl.), 35-59.

DECHOW, P.A., WEILI, G. \& SCHRAND, C. (2010): "Understanding earnings quality: A review of the proxies, their determinants and their consequences", Journal of Accounting and Economics, 50, 344-401.

DEFOND, M., HUNG, M. \& TREZEVANT, R. (2007): "Investor protection and the information content of annual earnings announcements: International evidence", Journal of Accounting and Economics, 43, 37-67.

DEMSKI, J. (1998): "Performance measure manipulation", Contemporary Accounting Research, 15, 261-285.

FRANCIS, J., LAFOND, R., OLSSON, P.M. \& SCHIPPER, K. (2004): "Cost of equity and earnings attributes", Accounting Review, 79, 967-1010.

FRANCIS, J. \& SMITH, M. (2005): "A reexamination of the persistence of accruals and cash flows," Journal of Accounting Research, 43(3), 413-451.

FRANCIS, J., LAFOND, R., OLSSON, P.M. \& SCHIPPER, K. (2005): "The market pricing of accrual quality", Journal of Accounting and Economics, 39, 295-327.

GABAS TRIGO, F. \& PINA MARTÍNEZ, V. (1991): "Alisamiento de los beneficios en el sector eléctrico: un estudio empírico", Revista española de financiación y contabilidad, 66, 265-283.

GALDEANO GÓMEZ, E. \& RODRÍGUEZ RODRÍGUEZ, M.C. (2000): "Cambios de estrategia en las entidades asociativas de comercialización hortofrutícola", Estudios Agrosociales y Pesqueros, 186, 75-103.

GARCÍA OSMA, B., GILL DE ALBORNOZ, B. \& GISBERT, A. (2005): "La investigación sobre earnings management", Revista Española de Financiación y Contabilidad, 34 (127), 1001-1033. 
GARCÍA OSMA, B. \& GILL DE ALBORNOZ, B. (2007): "The effect of the board composition and its monitoring committees on earnings management: Evidence from Spain", Corporate Governance, 15(6), 1412-1427.

GILL DE ALBORNOZ NOGUER, B. \& ILLUECA MUÑOZ, M. (2007): "La calidad de los ajustes por devengo no afecta al coste de la deuda de las pymes españolas", Investigaciones Económicas, XXXI (1), 79-117.

HEALY, P.M. \& PALEPU, K.G. (2001): "Information asymmetry, corporate disclosure, and the capital markets: A review of the empirical disclosure literature", Journal of Accounting and Economics, 31, 405-440.

ÍNIGUEZ SÁNCHEZ, R. \& POVEDA FUENTES, F. (2008): "Persistencia del resultado contable y sus componentes: implicaciones de la medida de ajustes por devengo", Revista española de financiación y contabilidad, XXXVII (137), 33-61.

JENSEN, M.C. \& MECKLING, W.H. (1976): "Theory of the firm: Managerial behavior, agency costs and ownership structure", Journal of Financial Economics, 3, 305-360.

JONES, J.J. (1991): "Earnings management during import relief investigation", Journal of Accounting Research, 29(2), 193-228.

JULIA, J. \& SERVER, R. (2003): "Social economy companies in the Spanish agricultural sector: delimitation and situation in the context of European Union", Annals of Public and Cooperative Economics, 74(3), 465-488.

JULIÁ, J. \& MELIÁ, E. (2008): "Social economy and de cooperative movement in Europe: input to a new vision of agriculture and rural development in the Europe of the 25", CIRIEC-España, Revista de Economía Pública, Social y Cooperativa, 62, 147-172.

KOTHARI, S.P., LEONE, A.J. \& WASLEY, C.E. (2005): "Performance matched discretionary accrual measures", Journal of Accounting \& Economics, 39(1), 163-197.

LARRAN JORGE M. (1997): "Un análisis crítico de la literatura empírica relativa a la contribución de la tesorería generada por las operaciones a la evaluación de la calidad del resultado", Revista española de financiación y contabilidad, XXVI (91), 387-425.

LARRAN JORGE, M. (2000): "Una revisión de la literatura empírica relativa a la utilidad del principio de caja: especial atención a la predicción del fracaso empresarial", Actualidad Financiera, 3, 33-60.

LEUZ, C., NANDA, D. \& WYSOCKI, P. (2003): "Earnings management and investor protection: An international comparison", Journal of Financial Economics, 69, 505-527.

LEV, B. (1983): "Some economic determinants of the timeseries properties of earnings", Journal of Accounting and Economics, 5, 31-38.

MCNICHOLS, M. (2002): "Discussion of the quality of accruals and earnings: The role of accrual estimation errors", Accounting Review, 77 (Suppl.), 61-69. 
MONTERREY MAYORAL, J. \& SÁNCHEZ-SEGURA, A. (2008): “Corporate governance and earnings quality: evidence from Spanish companies", RC-SAR, 11 (1), 67-100.

MONTERREY MAYORAL, J. \& SÁNCHEZ-SEGURA, A. (2009): "How taxes can affect earnings quality? Evidence from Spanish private firms", RC-SAR, 12 (1), 117-140.

NILSSON, J. (1999): "Co-operative organizational models as reflections of de business environments", Finnish Journal of Business Environments, 4, 449-470.

ORDEN EHA 3360/2010, de 21 de diciembre, por el que se aprueban las normas contables sobre aspectos contables de las sociedades cooperativas (BOE n 316, de 29 de diciembre de 2010)

PENMAN, S.H. \& ZHANG, X.J. (2002): "Accounting conservatism, the quality of earnings, and stock returns", Accounting Review, 77, 237-264.

PINEDA GONZÁLEZ, C. (2000): "Determinantes de la calidad del resultado", Revista de Contabilidad", $3(5), 149-182$.

REAL DECRETO 1514/2007, de 16 de noviembre, por el que se aprueba el Plan General de Contabilidad (BOE n 278, de 20 de noviembre).

REVSINE, D.W., COLLINS, D.W. \& JOHNSON, W.B. (2002): Financial reporting and analysis, 3rd ed. Upper Saddle River, NJ: Prentice Hall.

ROMÁN CERVANTES, C. (2008): "Las sociedades agrarias de transformación en España: un análisis histórico", CIRIEC-España, Revista de Economía Pública, Social y Cooperativa, 63, 65-87.

RONEN, J. \& SADAN, S. (1981): Smoothing income numbers: Objectives, means and implications, Reading, MA: Addison- Wesley.

SCHIPPER, K. \& VINCENT, L. (2003): "Earnings quality”, Accounting Horizons, 17 (Suppl.), 97-110.

SLOAN, R.G. (1996): "Do stock prices fully reflect information in accruals and cash flows about future earnings?", The Accounting Review, 71, 289-315.

VARGAS VASSEROT, C. \& AGUILAR RUBIO, M. (2004): "Las operaciones de la cooperativa con terceros y la infundada limitación de las mismas por su tratamiento fiscal privilegiado", REVESCO, Revista de Estudios Cooperativos, 83, 115-140.

VARGAS VASSEROT, C. (2010): "Las sociedades agrarias de transformación en España. Defectos legales y ventajas operativas", Estudios Agrarios, 2010, 75-107.

VICENT CHULIÁ, F. (2002): "El futuro de la legislación cooperativa", CIRIEC-España, Revista Jurídica de Economía Social y Cooperativa, 13, 9-48.

VIDAL, F., SEGURA, J.A. \& RAYOS, J.A. (2001): "Situación económico-financiera de las SAT de comercialización hortofrutícola de la provincia de Alicante", Invest. Agr.: Prod. Veg., 16(1), 72-86.

WATTS, R. \& ZIMMERMAN, J. (1986): Positive accounting theory. Englewood Cliffs, NJ: Prentice Hall. 